\title{
Alignment of the LHCb tracking system
}

13th ICATPP Conference on Astroparticle, Particle, Space Physics and Detectors for Physics Applications

\section{R. Märki for the LHCb alignment group}

\author{
EPFL - LPHE
}

03 October 2011 


\section{Outline}

- The software alignment procedure

- Introduction

- $\chi^{2}$ minimization

- Data samples and useful constraints

- Internal VELO alignment

- Presentation

- Features

- Performance

- Global alignment

- Presentation

- Features

- Performance

- Summary

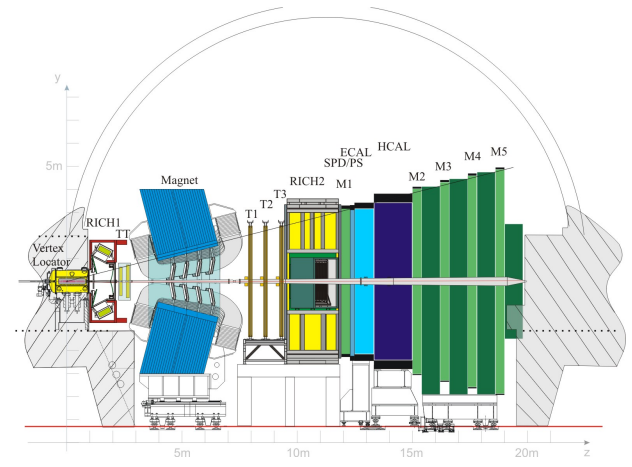




\section{What is software alignment?}

- All tracking subdetectors have been surveyed after assembly and installation

- Real tracks are used to know the position of the elements with an even higher precision

- This is called software alignment

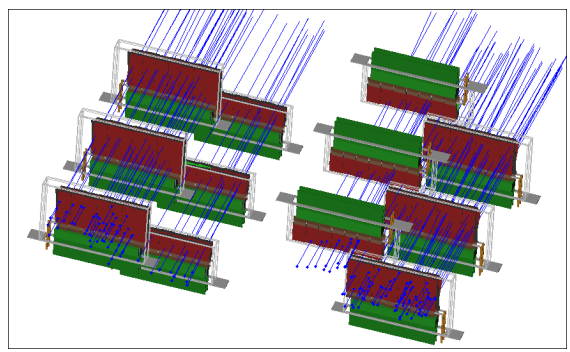




\section{Software alignment using tracks fitted with a Kalman filter}

Sample of selected good quality tracks

- $\chi^{2}$ calculated from track fit residuals

- The $\chi^{2}$ also gets a contribution from survey residuals

- Algorithm to minimize $\chi^{2}$ as a function of alignment parameters

$$
\chi^{2}=\sum_{\text {hits } i}\left(\frac{m_{i}-h_{i}(x)}{\sigma_{i}}\right)^{2}
$$

$\mathrm{m} \rightarrow$ measurement, $\sigma \rightarrow$ measurement error

$x \rightarrow$ track parameters, usually 5

$\mathrm{h} \rightarrow$ measurement model

$$
0 \equiv \frac{d \chi^{2}}{d x}
$$

*W. Hulsbergen, The global covariance matrix of tracks fitted with a Kalman filter and an application in detector alignment, Nucl. Instr. and Meth. A, 600 (2009), p. 471. 


\section{Degrees of freedom and weak modes}

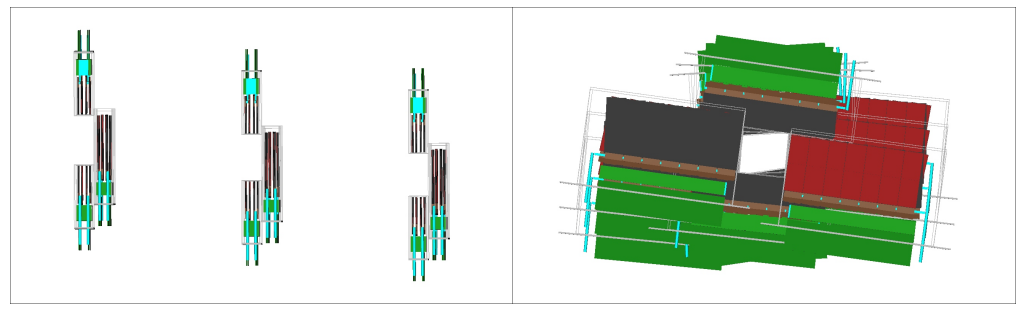

- Chose degrees of freedom (alignment variables)

- translations and rotations

- Degrees of freedom are differently chosen at all levels

- whole detector, layer, sensor, etc.

- Low sensitivity for certain degrees: weak modes

- eg. translations along beam axis, scaling, shearing, ... 


\section{Data for alignment - find the best constraints}
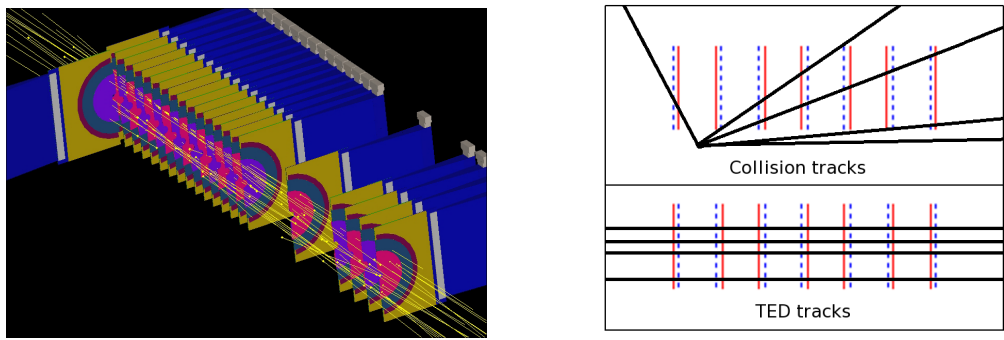

Different type of data giving different constraints

- Tracks from TED runs (collisions far away from the detector) and proton-beam gas collisions

$\rightarrow$ small angle tracks

- Collisions - happening within the VELO

$\rightarrow$ various angle tracks

Also very important constraints

- Overlaps between subdetectors (eg. IT and OT)

- Overlaps between sensors within a subdetector

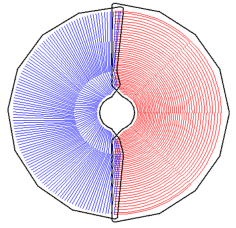

VELO fully closed (stable beam) 


\section{VELO alignment - metrology and track alignment}

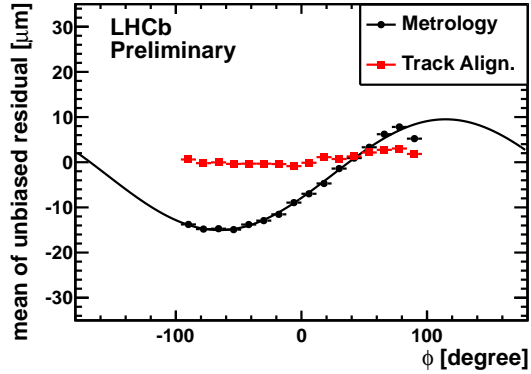

$\mathrm{R}$ sensor number 38
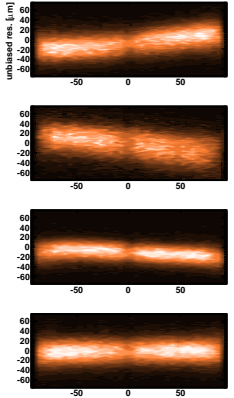

Metrology (left) / Alignmnent (right)

res. $(\mathrm{R})=-\Delta x \cos \phi_{\text {track }}+\Delta y \sin \phi_{\text {track }}$

res. $(\phi)=\Delta x \sin \phi_{\text {track }}+\Delta y \cos \phi_{\text {track }}+\Delta \gamma r_{\text {track }}$

- Initially used Millepede alignment starting from metrology

- Now $\chi^{2}$ minimization of Kalman fit residuals

- Work on Z (beam axis) position ongoing (low sensitivity due to weak modes) - for the moment use metrology 


\section{VELO alignment and primary vertices}
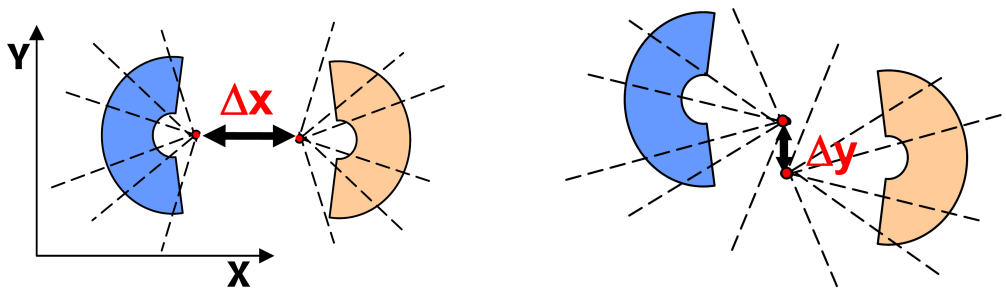

PV left-right method

- Compute primary vertex with each half of the detector

- Calculate average distance between left and right PVs

$\rightarrow$ two half misalignment

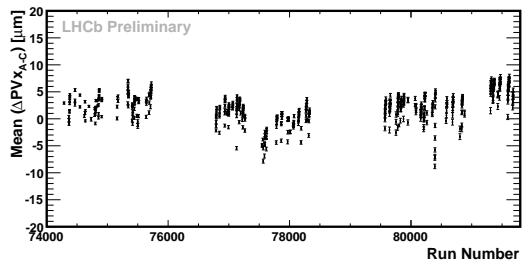

Also done in the software alignment

- Use particularly primary vertices to align the VELO halves

- Add overlap tracks to "link" the two halves 


\section{VELO alignment with beam-gas events}

Mean of $\mathrm{IP}_{\mathrm{X}} \mathrm{vs} \phi[\mathrm{rad}]$

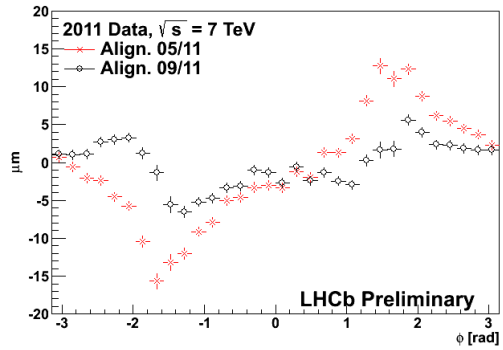

Mean of $I P_{Y}$ vs $\phi[\mathrm{rad}]$

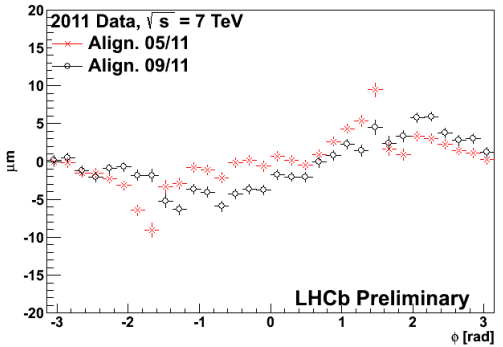

- Main goal: reduce Rz weak mode

- Use beam-gas selection (including standard collisions and satellite collisions at $\pm 700 \mathrm{~mm}$ around the nominal collision point)

- In these events: take tracks which cross many sensors (many tracks from the satellite collisions cross the full VELO)

- Align for all degrees of freedom

- Validate with collision data

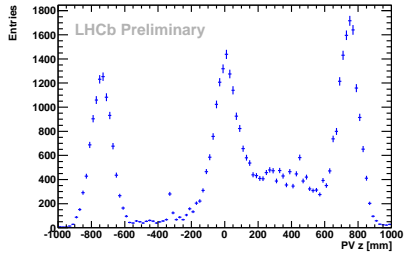




\section{VELO alignment performance}
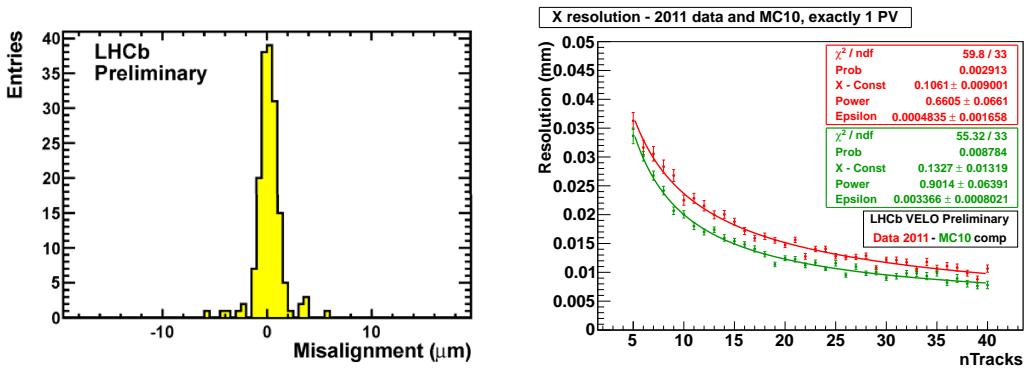

- Sensor alignment better than $4 \mu \mathrm{m}$

- PV resolution $(x, y, z)$ with 25 tracks:

- Data $(13.0,12.5,68.5) \mu \mathrm{m}$

- $\operatorname{MC}(10.7,10.9,58.1) \mu \mathrm{m}$

- Still room for improvement

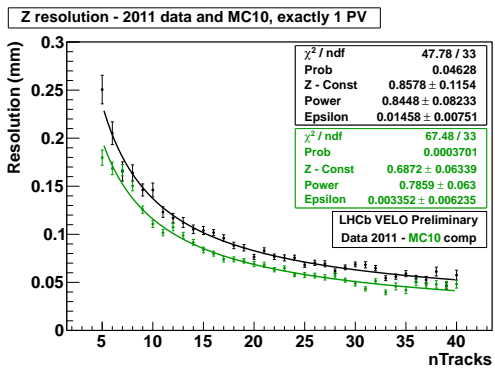




\section{Global alignment}

- TT, IT and OT are aligned by $\chi^{2}$ minimization all at once

- Mostly tracks crossing the whole detector are chosen

- Additional kinematic cuts are applied

- high momentum, high angle, good quality, etc.

- Some elements stay fixed along Z to survey constants

- Whole TT subdetector

- Two layers in OT

$\rightarrow$ constrain the global position of IT and OT
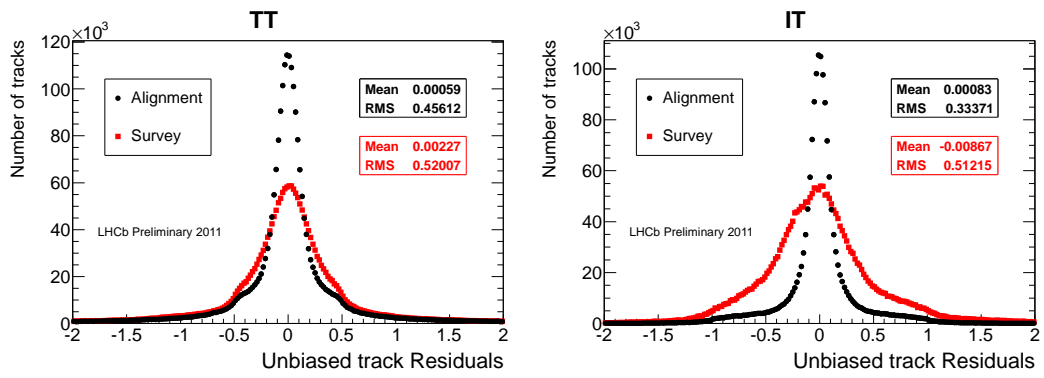


\section{Global alignment - Mass constraint}
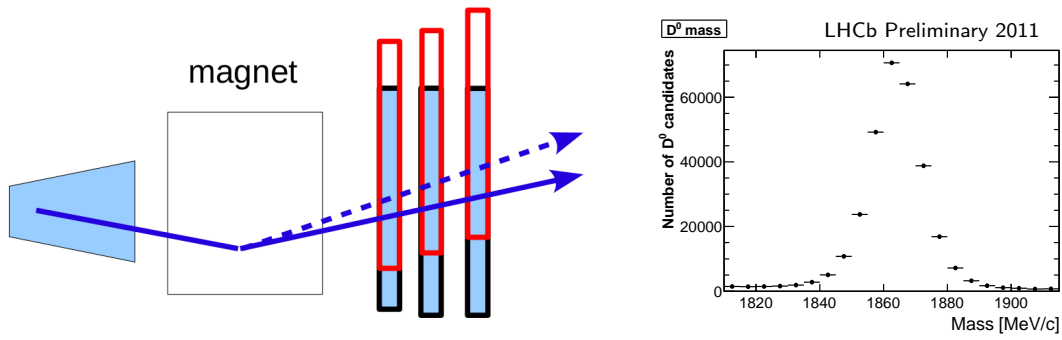

Weak mode: shearing with respect to magnet axis. Therefore mass constrained alignment (J/Psi or D0)

- Take $J / \psi \rightarrow \mu \mu$ or $D^{0} \rightarrow K \pi$ daughter tracks

- Fix their origin vertex

- Fix the invariant mass to known $\mathrm{J} / \psi$ or $D^{0}$ mass respectively

- Compute the new tracks + residuals which go into the $\chi^{2}$ minimization 


\section{Global alignment - $Y$ alignment}

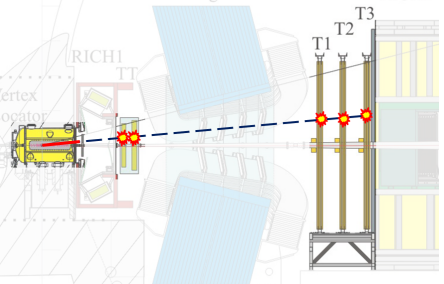

IT and TT $Y$ alignment method

- Extrapolate VELO tracks to IT or TT in events without magnetic field

- Search for corresponding hits

- Find gaps and edges in their $Y$ distribution
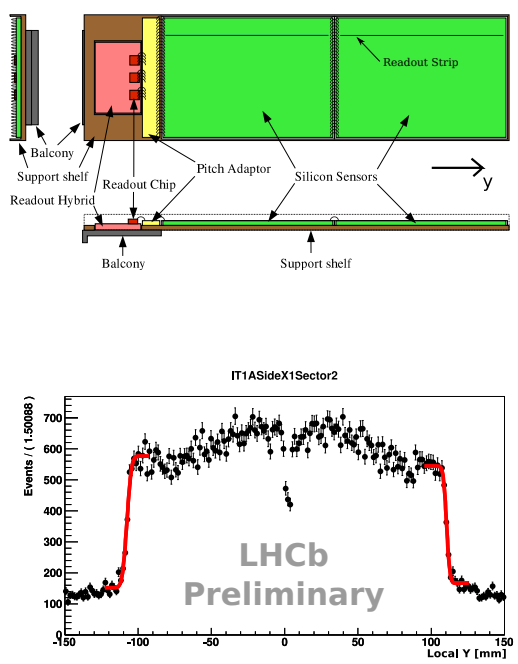


\section{Global alignment performance}

IT Sector Biased Residuals

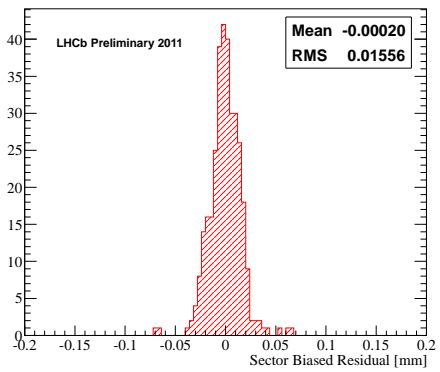

TT Sector Biased Residuals

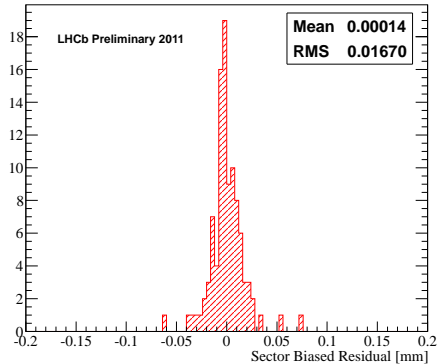

- Alignment precision can be evaluated by residual bias

- IT Misalignment $15.6 \mu \mathrm{m}$

- TT Misalignment $16.7 \mu \mathrm{m}$

- Even small errors in geometry implementation found by alignment

- Here also room for improvement 


\section{Summary}

- Alignment not sensitive to all degrees of freedom, eg. Z position often fixed to survey constants

- Various methods to improve alignment - counter some weak-modes

- Align VELO with primary vertices and overlap tracks

- Use TED and beam-gas events and satellite collisions

- Use masses of known particles as constraint

- Extrapolate tracks from data without magnetic field

- Silicon strip detectors: sensor position known with a precision of

- $4 \mu \mathrm{m}$ for VELO

- $15.6 \mu \mathrm{m}$ for IT

- $16.7 \mu \mathrm{m}$ for TT 


\section{BACKUP SLIDES}




\section{The LHCb tracking system}

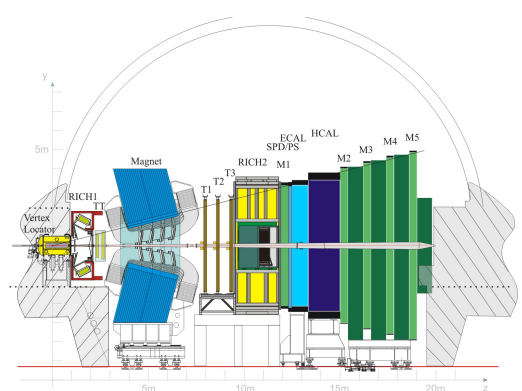

- VErtex LOcator (VELO)

- Silicon strip detector, closest to the collision point

- Tracker Turicensis (TT)

- Silicon strip detector, between the VELO and the magnet

- Inner Tracker (IT)

- Silicon strip detector, after the magnet, covering the innermost part

- Outer Tracker (OT)

- Straw tube drift chambers, after the magnet, covering the outer part 